\title{
Hydrodynamic Modeling of Tissue Ablation with a Free- electron Laser
}

R.A. London, A.J. Makarewicz, S.R. Uhlhorn, E.D. Jansen, S.R. Uhlhorn

This article was submitted to

Lasers Tissue Interaction XI, Bellingham, WA, January 22-26, 2000

U.S. Department of Energy

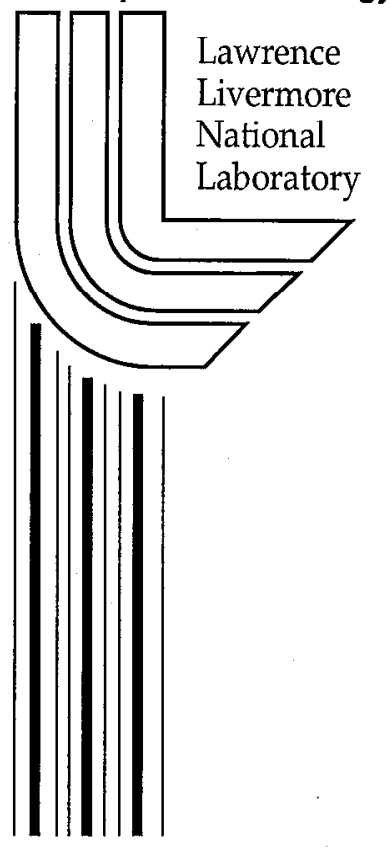

February 24, 2000 


\section{DISCLAIMER}

This document was prepared as an account of work sponsored by an agency of the United States Government. Neither the United States Government nor the University of California nor any of their employees, makes any warranty, express or implied, or assumes any legal liability or responsibility for the accuracy, completeness, or usefulness of any information, apparatus, product, or process disclosed, or represents that its use would not infringe privately owned rights. Reference herein to any specific commercial product, process, or service by trade name, trademark, manufacturer, or otherwise, does not necessarily constitute or imply its endorsement, recommendation, or favoring by the United States Government or the University of California. The views and opinions of authors expressed herein do not necessarily state or reflect those of the United States Government or the University of California, and shall not be used for advertising or product endorsement purposes.

This is a preprint of a paper intended for publication in a journal or proceedings. Since changes may be made before publication, this preprint is made available with the understanding that it will not be cited or reproduced without the permission of the author.

This report has been reproduced

directly from the best available copy.

Available to DOE and DOE contractors from the

Office of Scientific and Technical Information

P.O. Box 62, Oak Ridge, TN 37831

Prices available from (423) 576-8401

http://apollo.osti.gov/bridge/

Available to the public from the

National Technical Information Service

U.S. Department of Commerce

5285 Port Royal Rd.,

Springfield, VA 22161

http://www.ntis.gov/

OR

Lawrence Livermore National Laboratory

Technical Information Department's Digital Library

http://www.llnl.gov/tid/Library.html 


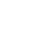




\title{
Hydrodynamic modeling of tissue ablation with a free-electron laser
}

\author{
Stephen R. Uhlhorn ${ }^{a, b}$, Richard A. London ${ }^{b}$, Anthony J. Makarewicz ${ }^{b}$, and E. Duco Jansen ${ }^{a}$ \\ ${ }^{a}$ Vanderbilt University, Department of Biomedical Engineering \\ Box 351631, Station B, Nashville, TN 37235, USA \\ ${ }^{b}$ Lawrence Livermore National Laboratory \\ P.O. Box 808, Livermore, CA 94551, USA
}

\begin{abstract}
The Vanderbilt University free-electron laser (FEL) provides a continuously tunable $(\lambda=2-10 \mu \mathrm{m})$ source of pulsed IR radiation with a pulse structure unlike those of conventional lasers (a macropulse of $5 \mu$ s consisting of a train of 1 ps micropulses at a frequency of $3 \mathrm{GHz}$ ). A numerical hydrodynamic code at Lawrence Livermore National Laboratory, known as LASTIS3D, was used to model the ablation of tissue using the FEL. This study investigates the role of the FEL pulse structure by comparing the results from simulations using a time-averaged energy deposition and a pulsetrain energy deposition.
\end{abstract}

Keywords: free electron laser, tissue ablation, hydrodynamic modeling

\section{INTRODUCTION}

The Vanderbilt University free electron laser is a continuously tunable source of pulsed mid-IR radiation with a wavelength range of $\lambda=2-10 \mu \mathrm{m}$. Previous studies have suggested that by tuning the FEL to the vibrational mode of the amide-II bonds in protein $(\lambda=6.45 \mu \mathrm{m})$ the FEL is well suited for precise tissue cutting and ablation with minimal collateral damage. ${ }^{1}$ This original study has been followed by several investigations into the role of the FEL wavelength in tissue ablation ${ }^{2-5}$ however, none of these studies have considered the effect of the FEL pulse structure in tissue ablation. Previously reported ${ }^{6}$ experimental results have been largely inconclusive, in part due to the ultrashort duration of the micropulses.

A diagram of the pulse structure of the FEL is seen in Figure 1. It consists of a macropulse of 4-6 $\mu$ s at a repetition rate of up to $30 \mathrm{~Hz}$. The macropulse is the envelope of a high frequency ( $3 \mathrm{GHz}$ ) train of micropulses with a duration of about $1 \mathrm{ps}$, separated by approximately $350 \mathrm{ps}$. This pulse structure results in a laser that is capable of both high peak $(10 \mathrm{MW})$ and average $(100 \mathrm{~kW})$ powers. If a laser pulse is shorter than the time necessary for heat to diffuse out of an irradiated volume, it is said to be thermally confined. Likewise, if the pulse is shorter than the time nccessary for a stress wave to propagate out of the irradiated volume, it is said to be inertially confined. A diagram of these confinement conditions for many common laser systems appears in Figure 2. In it, the penetration depth $\delta$ is plotted against the pulse duration $\tau_{L}$ for the pulses of some common laser systems. The condition for thermal confinement is given by the line $\tau=\delta^{2} / 4 \alpha$, where $\alpha$ is the thermal diffusivity of the medium. As the pulse duration is further decreased, the condition for inertial confinement given by the line $\tau=\delta / \sigma$, where $\sigma$ is the speed of sound in the medium. It can be seen from Figure 2 that the FEL macropulse is thermally confined at all penetration depths except those below $\approx 1 \mu \mathrm{m}$, which is only seen at wavelengths near the strong water absorption peak at $\lambda=3.0 \mu \mathrm{m}$. Further, both the FEL micropulses (1 ps) and the micropulse separation (350 ps) are short compared to the inertial confinement time for all penetration depths. This raises the possibility that large amplitude stress waves may be generated by the FEL micropulse structure.

In order to investigate the effects of the micropulse structure on a sufficiently short time scale, we turned to theoretical modeling using a numerical hydrodynamic code called LATIS3D that is being developed at Lawrence Livermore National Laboratory (LLNL). The current study investigated the effect of the FEL micropulse structure on the laser-induced stress waves. We hypothesize that the results of ablation with the FEL can not be solely attributed to wavelength tunability and specific absorption in amide bands.

Further author information: (Send correspondence to S.R.U.) S.R.U.: E-mail: stephen.uhlhorn@vanderbilt.edu 


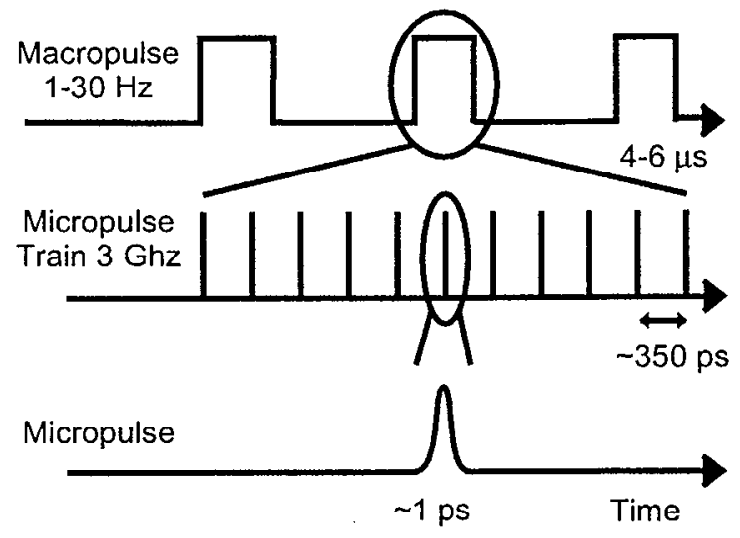

Figure 1. Pulse structure of the FET.

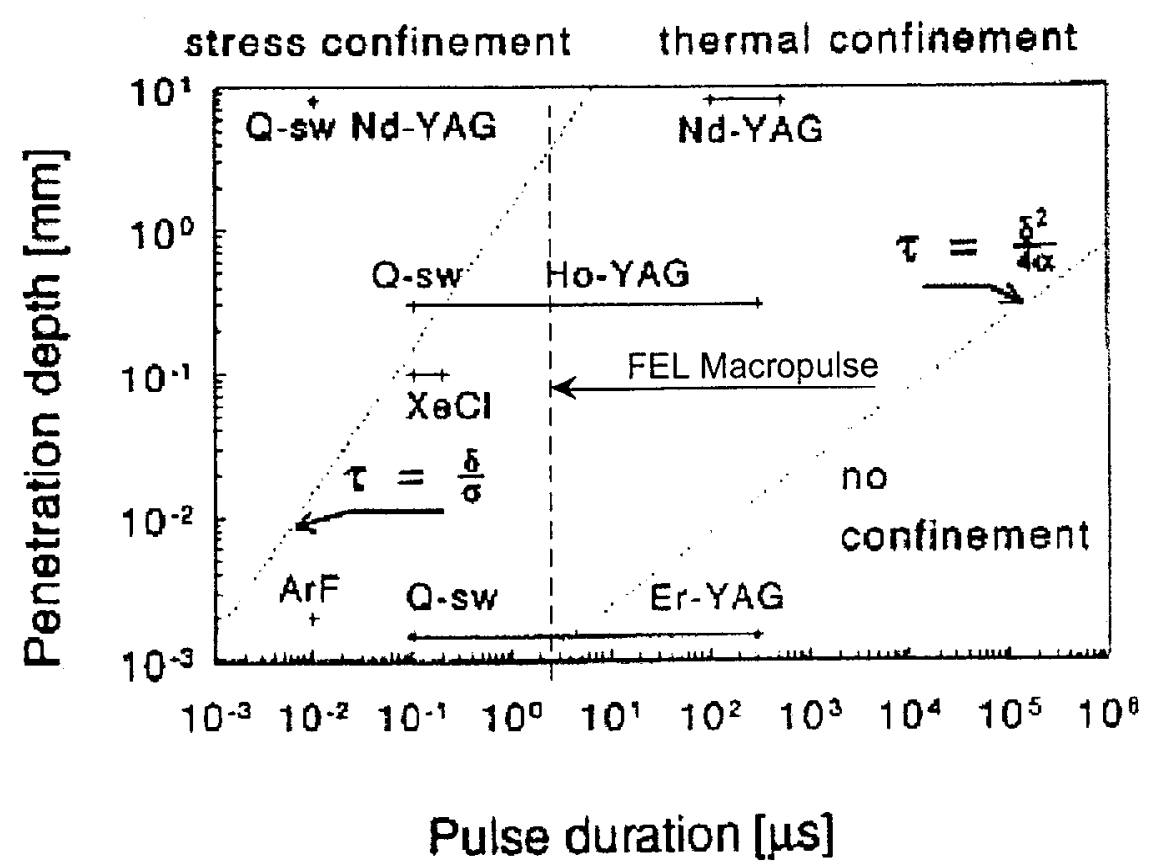

Figure 2. Confinement regions as a function of pulse duration for various laser pulses including the FEL macropulse. Modified from Welch et al. ${ }^{7}$ 


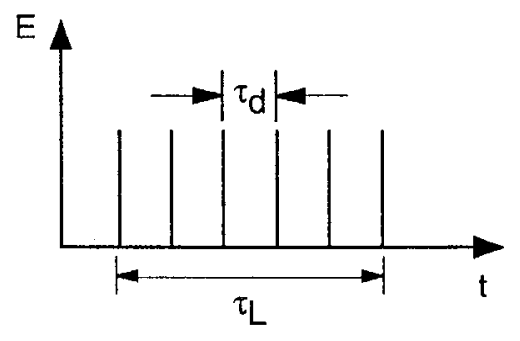

A

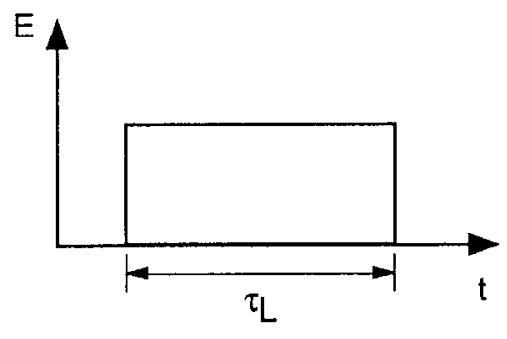

B

Figure 3. Time-dependent energy depostion schemes for the model simulations. A) pulsetrain and B) time-averaged deposition. $\tau_{\mathrm{L}}$ is the laser pulse duration and $\tau_{\mathrm{d}}$ is the delay between micropulses.

\section{MODELING METHODS}

LATIS3D is a subset of Kull, a larger simulation code at LLNL. Kull is a comprehensive physics package, based on finite volumes and written in the computer language $\mathrm{C}++$, that includes light transport, fluid flow, materials response, and heat transfer modeling. The current study only considers the hydrodynamic response of the material, which is calculated using the standard thermodynamic conservation equation for mass, momentum, and energy:

$$
\begin{gathered}
\frac{\partial \rho}{\partial t}=-\rho \vec{\nabla} \cdot \vec{v}, \\
\frac{\partial \rho v^{k}}{\partial t}=-\frac{\partial P}{\partial x^{k}}-\rho v^{k} \vec{\nabla} \cdot \vec{v}+\rho \vec{g}_{k}, \\
\frac{\partial \rho \varepsilon}{\partial t}=-P \vec{\nabla} \cdot \vec{v}-\rho \varepsilon \vec{\nabla} \cdot \vec{v} .
\end{gathered}
$$

We used a tabular equation of state of water to model the tissue and only sub-threshold fluences were initially used. The stress waves in the medium are generated by a time-dependent energy source deposition with a Beer's Law absorption distribution. Only one dimensional propagation is considered by making the laser spot radius $r$ much larger than the penetration depth $\delta$. Two types of laser irradiation schemes were considered: 1) a pulsetrain energy deposition that is similar the pulse structure of th FEL and 2) a time-averaged energy deposition that had an equivalent total energy as the pulsetrain, but delivered at a constant power level. These two pulse deposition schemes can be seen in Figure 3. For both cases, the laser spot radius $r=100 \mu \mathrm{m}$, and the penetration depth $\delta=10 \mu \mathrm{m}$, which is approximately the penetration depth of water at $\lambda=6.45 \mu \mathrm{m}$. The total energy delivered to the sample in both the pulsetrain deposition (Figure 3a) and the time-averaged deposition (Figure 3b) was $\mathrm{E}_{\mathrm{tot}}=50 \mu \mathrm{J}$ which was deposited over the same pulse duration $\tau_{\mathrm{L}}=50 \mathrm{~ns}$. For the pulsetrain deposition, it is assumed that the individual micropulse are absorbed instantaneously, that is, the micropulse duration is short compared to the inertial confinement time. The energy contained within one micropulse was $\mathrm{E}=0.3 \mu \mathrm{J}$, with a delay between micropulses $\tau_{\mathrm{d}}=333 \mathrm{ps}$ and a total of $\mathrm{n}=150$ pulses delivered. The numerical mesh consisted of 20 zones $/ \mu \mathrm{m}$.

\section{RESULTS}

The results from the model simulations appcar in Figurcs 4, 5, and 6. Figure ?? shows the stress waved generated from both the pulsetrain and time-averaged energy deposition described in section 2 . For both schemes, we see that the peak stress amplitudes are comparable, however in the pulsetrain deposition, it appears that there is a high frequency noise imposed on the stress waves. By looking at an expanded scale (Figure 5), we can see that the high frequency noise is well correlated with the pulsetrain. There are 150 pressure transients present and time delay between them is exactly the same as the delay between subsequent pulses (333 ps).

This correlation between the pressure transients and the delivered pulses is more clearly seen in Figure 6 . In it, the total energy was the same as the previous simulations $\left(E_{\mathrm{tot}}=50 \mu \mathrm{J}\right)$ which was delivered on over the same duration $\left(\tau_{\mathrm{L}}=50 \mu \mathrm{s}\right)$. However, the number of pulses was reduced to $\mathrm{n}=50$ and the delay between pulses was increased to $\tau=1 \mathrm{~ns}$. Again, we see that the pressure transients are well correlated with the pulses delivered, both in the total number (50) and in the delay between each pulse (1 ns). 

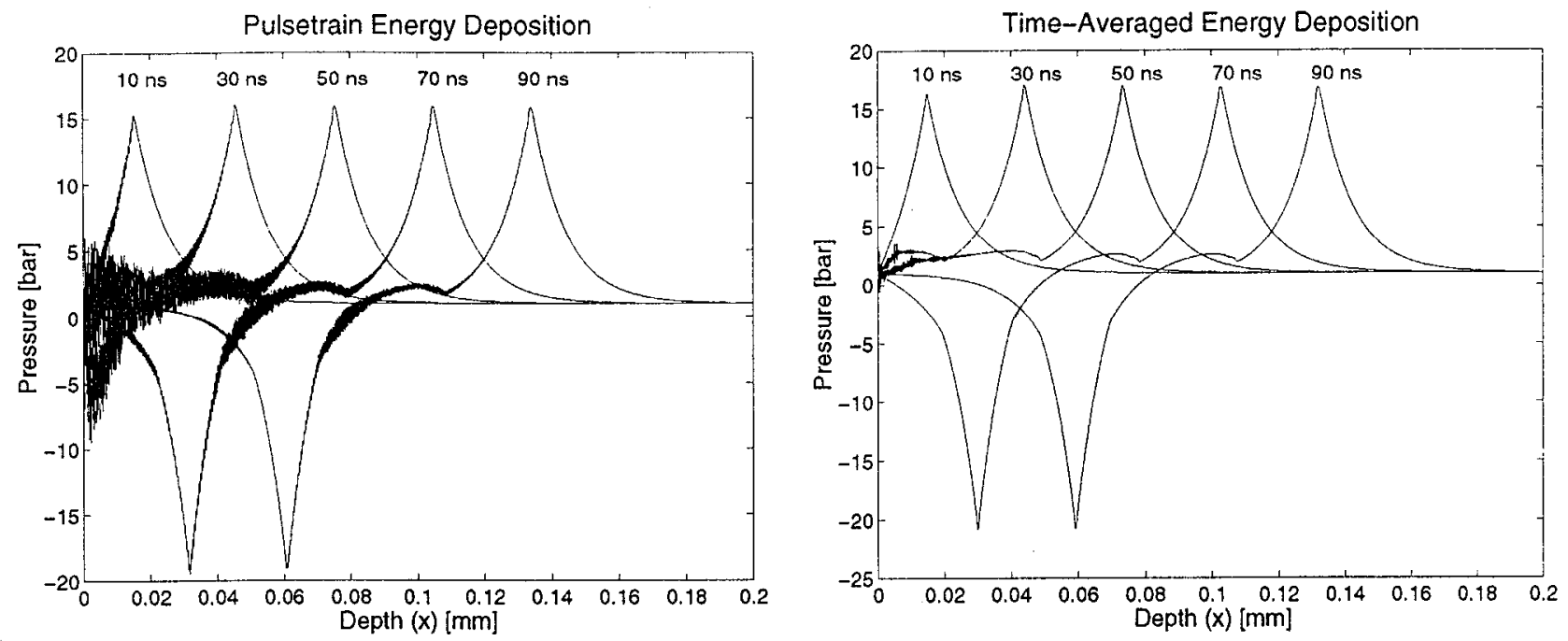

Figure 4. Pressure amplitude as a function of tissue depth at multiple times for both the pulsetrain and timeaveraged energy deposition schemes. The numerical grid is $200 \mu \mathrm{m}$ deep.
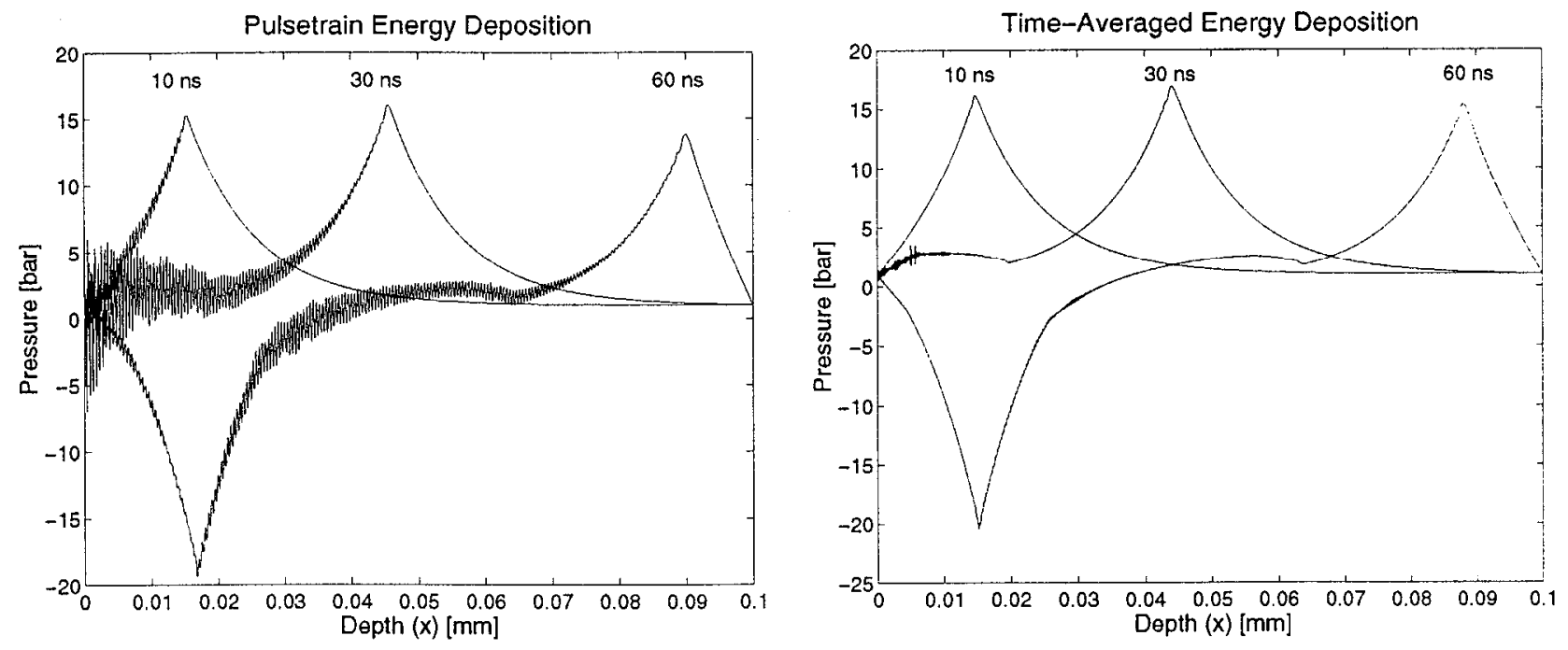

Figure 5. Pressure amplitude as a function of tissue depth at multiple times for both the pulsetrain and timeaveraged energy deposition schemes. The numerical grid is $100 \mu \mathrm{m}$ deep. 


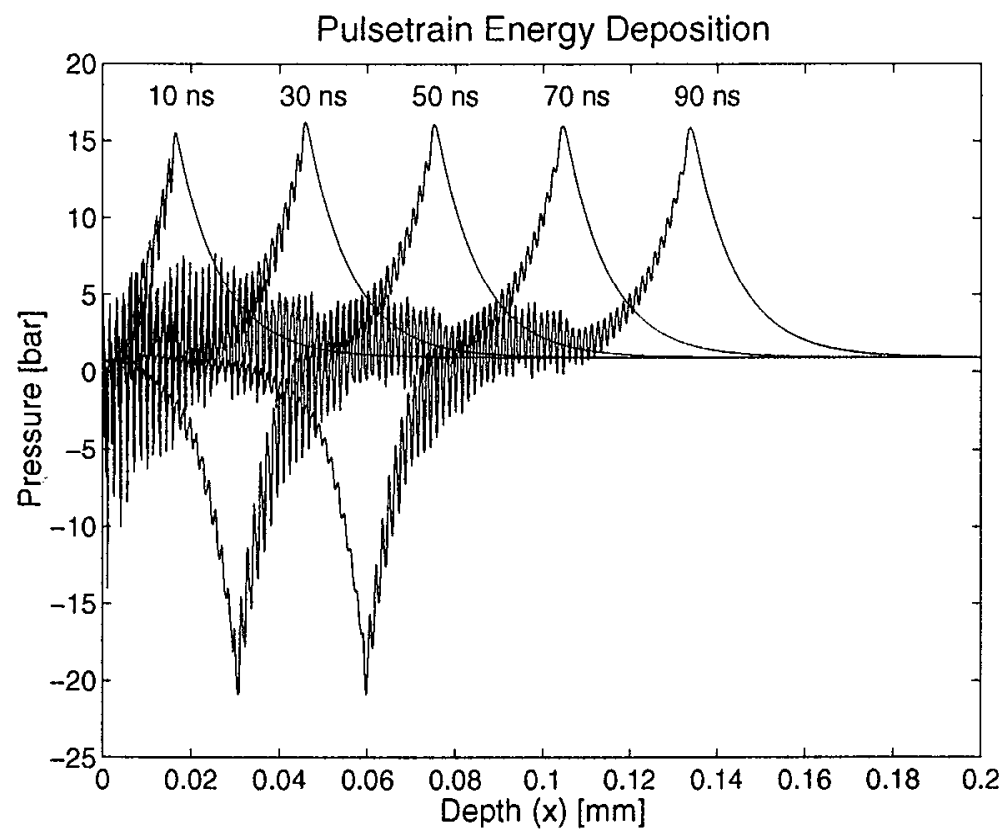

Figure 6. Pressure amplitude as a function of tissue depth at multiple times for the pulsetrain energy deposition scheme. The numerical grid is $200 \mu \mathrm{m}$ deep.

\section{DISCUSSION}

In comparing the results from the pulsetrain and time-averaged energy depositions, we see that the peak stress wave amplitudes generated are the same, which is exactly what one would expect given that the average radiant exposure $\left(E_{\mathrm{tot}} / \tau_{\mathrm{L}}\right)$ is constant for both cases. However, for the pulsetrain deposition, we see that large local pressure transients are generated. These local pressure transients create large pressure gradients that are on the order of $\frac{d P}{d x}=300 \mathrm{bar} / \mu \mathrm{m}$. Such large pressure gradients, which have both a compressive and tensile component, may effect tissue on a cellular level by tearing cell membranes and other microstructures.

The data that has been presented shows that the micropulse structure has an effect on the stress waves that are generated by laser exposure. This suggests that the results of ablation with the FEL can not be solely attributed to wavelength its tunability and the selective absorption of multiple tissue components.

\section{ACKNOWLEDGMENTS}

This work was performed under the anspices of the U.S. Department of Energy by Lawrence Livermore National Laboratory under contract W-7405-ENG-48.

\section{REFERENCES}

1. G. Edwards, R. Logan, M. Copeland, L. Reinisch, J. Davidson, B. Johnson, R. Maciunas, M. Mendenhall, R. Ossoff, J. Tribble, J. Werkhaven, and D. O'Day, "Tissue ablation by a free-electron laser tuned to the amide II band," Nature 371, pp. 416-419, 1994.

2. D. L. Ellis, N. K. Weisberg, J. S. Chen, G. P. Stricklin, and L. Reinisch, "Free electron laser wavelength specificity for cutaneous contraction," Lasers in Surgery and Medicine 25, pp. 1-7, 1999.

3. J. M. Auerhammer, R. Walker, A. F. G. van der Meer, and B. Jean, "Dynamic behavior of photoablation products of corneal tissue in the mid-ir: a study with felix," Applied Physics B 68, pp. 111-119, 1999.

4. M. Ostertag, J. T. McKinley, L. Reinisch, D. M. Harris, and N. H. Tolk, "Laser ablation as a function of the primary absorber in dentin," Lasers in Surgery and Medicine 21, pp. 384-394, 1997.

5. J. Tribble, D. C. Lamb, L. Reinisch, and G. Edwards, "Dynamics of gelatin ablation due to free-electron-laser irradiation," Physical Review E 55(6), pp. 7385-7389, 1997. 
6. S. R. Uhlhorn, S. Harrison, H. S. Preatisto, and E. D. Jansen, "Tissue ablation with the free-electron laser: Contributions of wavelength and pulse structure," in Laser-Tissue Interaction X, SPIE, 1999.

7. A. J. Welch and M. J. C. van Gemert, eds., Optical-Thermal Response of Laser-Irradiated Tissue, ch. 21, pp. 709763. Plenum Press, New York, 1995. 